Aggregate von vorgegebenen Potenzen, Arkiv för Matematik, Astronomi och Fysik 17 (1922-1923), no. 9, 1-30.

3. S. N. Mergelyan, Uniform approximation to functions of a complex variable, Amer. Math. Soc. Transl. 101 (1954), 2-99.

Columbia University,

UNIVERSITY OF ILLINOIS, AND

UNIVERSITY OF MICHIGAN

\title{
DOUBLY INVARIANT SUBSPACES OF ANNULUS OPERATORS
}

BY DONALD SARASON ${ }^{1}$

Communicated by P. R. Halmos, April 29, 1963

1. Introduction. Let $C$ be the unit circle in the complex plane and let $C_{0}$ be the circle $\left\{z:|z|=r_{0}\right\}$, where $r_{0}$ is a positive real number less than unity. The set $C \cup C_{0}$ is the boundary of the annulus $A=\left\{z: r_{0}<|z|<1\right\}$. Let us endow the circles $C$ and $C_{0}$ with Lebesgue measure of total mass unity, and denote by $L^{2}(\partial A)$ the $L^{2}$ space associated with the measure thereby defined on the set $C \cup C_{0}$. This note concerns the invariant subspaces of the position operator on the space $L^{2}(\partial A)$, that is, of the operator $Z$ on $L^{2}(\partial A)$ defined by $(Z x)(z)$ $=z x(z)$.

We may regard $L^{2}(\partial A)$ as the direct sum of the two spaces $L^{2}(C)$ and $L^{2}\left(C_{0}\right)$. As subspaces of $L^{2}(\partial A)$, the latter reduce the operator $Z$. The restriction of $Z$ to $L^{2}(C)$ is a well-known operator, a so-called bilateral shift (of unit multiplicity). The invariant subspaces of this operator have been extensively studied by Beurling [1], by Helson and Lowdenslager [3], and by Halmos [2]. The restriction of $Z$ to $L^{2}\left(C_{0}\right)$ is a bilateral shift multiplied by the scalar $r_{0}$, and so has the same invariant subspace structure as a bilateral shift. The operator $Z$ is therefore the direct sum of two operators whose invariant subspaces have been completely described. However, the problem of determining the invariant subspaces of $Z$ involves more than merely a routine extension of known results about bilateral shifts, and as yet has not been solved completely.

\footnotetext{
${ }^{1}$ Research supported in part by the National Science Foundation. The results announced in this paper constitute a portion of the author's University of Michigan Doctoral Dissertation. I am deeply indebted to Professor Paul Halmos for the help he has given me over the past year.
} 
The purpose of this note is to announce results concerning the invariant subspaces of $Z$ that are also invariant under $Z^{-1}$; these we call doubly invariant subspaces of $Z$. The proofs, to appear elsewhere, depend to a large extent on complex function theory, and involve analogues for functions in the $H^{2}$ space of the annulus $A$ of a number of well-known properties of functions in the $H^{2}$ space of a disk.

2. A characterization of doubly invariant subspaces. For every real number $\alpha$ we define the function $w_{\alpha}$ on $C \cup C_{0}$ by

$$
\left.\begin{array}{rl}
w_{\alpha}\left(e^{i t}\right) & =e^{i \alpha t} \\
w_{\alpha}\left(r_{0} e^{i t}\right) & =r_{0}^{\alpha} e^{i a t} t
\end{array}\right\} \quad 0 \leqq t<2 \pi .
$$

Then $Z w_{\alpha}=w_{\alpha+1}$, and the functions $w_{\alpha}$ and $w_{\alpha+n}$ are orthogonal for $n= \pm 1, \pm 2, \cdots$. For $0 \leqq \alpha<1$ we denote by $H_{\alpha}^{2}(\partial A)$ the smallest doubly invariant subspace of $Z$ containing $w_{\alpha}$, that is, the span in $L^{2}(\partial A)$ of the functions $w_{\alpha+n}, n=0, \pm 1, \pm 2, \cdots$. More generally, for any function $x$ in $L^{2}(\partial A)$ we let $M_{x}$ denote the smallest doubly invariant subspace of $Z$ containing $x$. The subspaces $H_{\alpha}^{2}(\partial A)$ are prototypes of the doubly invariant subspaces that do not reduce $Z$. More precisely, we have the following two theorems.

TheOREM 1. Let $x$ be a function in $L^{2}(\partial A)$. If the condition

$$
\int_{0}^{2 \pi} \log \left|x\left(e^{i t}\right)\right| d t+\int_{0}^{2 \pi} \log \left|x\left(r_{0} e^{i t}\right)\right| d t>-\infty
$$

is satisfied, let $\alpha$ be the number in the interval $[0,1)$ congruent modulo 1 to the number

$$
\frac{1}{2 \pi q_{0}}\left[\int_{0}^{2 \pi} \log \left|x\left(e^{i t}\right)\right| d t-\int_{0}^{2 \pi} \log \left|x\left(r_{0} e^{i t}\right)\right| d t\right],
$$

where $q_{0}=-\log r_{0}$. Then there is a measurable function w on $C \cup C_{0}$, with $|w|=1$ almost everywhere, such that $M_{x}$ consists of all products wy with $y$ in $H_{\alpha}^{2}(\partial A)$. The function w is unique to within a multiplicative constant of unit modulus.

On the other hand, if condition (*) is not satisfied (that is, if the function $x$ is "small"), then $M_{x}$ consists of all functions in $L^{2}(\partial A)$ that vanish at every point where $x$ vanishes.

THEOREM 2. If $M$ is any doubly invariant subspace of $Z$, then there is a function $x$ such that $M=M_{x}$.

Theorems 1 and 2 characterize the doubly invariant subspaces of $Z$. We see in particular that if $x$ is a function in $L^{2}(\partial A)$ satisfying $\left({ }^{*}\right)$, 
and if $\alpha$ is as defined in Theorem 1 , then the operator $Z \mid M_{x}$ is unitarily equivalent to $Z \mid H_{\alpha}^{2}(\partial A)$. On the other hand, one can show that the operators $Z \mid H_{\alpha}^{2}(\partial A)$ and $Z \mid H_{\beta}^{2}(\partial A)$ are not unitarily equivalent for $\alpha \neq \beta$.

3. Doubly invariant subspaces in $H^{2}(A)$. The space $H^{2}(A)$ consists by definition of all holomorphic functions $f$ in the annulus $A$ such that

$$
\sup _{r_{0}<r<1} \int_{0}^{2 \pi}\left|f\left(r e^{i t}\right)\right|^{2} d t<\infty .
$$

Just as in a disk, a function of class $H^{2}(A)$ has nontangential limits at almost every boundary point of $A$, and so can be extended (almost everywhere) to the boundary. By Fatou's lemma, the resulting boundary function belongs to $L^{2}(\partial A)$. In fact, one can show that the boundary function belongs to $H_{0}^{2}(\partial A)$. Conversely, any function in $H_{0}^{2}(\partial A)$ is the boundary function of a unique function in $H^{2}(A)$. The spaces $H_{0}^{2}(\partial A)$ and $H^{2}(A)$ are thus in one-to-one correspondence, and the latter is thereby endowed with a Hilbert space structure. The operator $Z \mid H_{0}^{2}(\partial A)$ corresponds to the operator $Z_{0}$ on $H^{2}(A)$ defined by $\left(Z_{0} f\right)(z)=z f(z)$. We conclude with two results concerning doubly invariant subspaces of the operator $Z_{0}$.

TheOREM 3. Let $a_{1}, a_{2}, a_{3}, \cdots$ be a finite or infinite sequence of points in the annulus $A$ (repetitions allowed). Let $M$ be the collection of all functions in $H^{2}(A)$ that vanish (with the appropriate multiplicity) at each point $a_{k}$. Then $M$ is a doubly invariant subspace of $Z_{0}$. If the sum

$$
\sum \min \left(1-\left|a_{k}\right|, 1-\frac{r_{0}}{\left|a_{k}\right|}\right)
$$

is infinite, then $M$ is trivial. If the sum (**) is finite, then $Z_{0} \mid M$ is unitarily equivalent to $Z \mid H_{\alpha}^{2}(\partial A)$, where $\alpha$ is the number in the interval $[0,1)$ congruent modulo 1 to the number $\sum \alpha_{k}$, the $\alpha_{k}$ being defined by

$$
\alpha_{k}= \begin{cases}\frac{-1}{q_{0}} \log \left|a_{k}\right| & \text { if } r_{0}^{1 / 2} \leqq\left|a_{k}\right|<1, \\ \frac{-1}{q_{0}} \log \left(\left|a_{k}\right| / r_{0}\right) & \text { if } r_{0}<\left|a_{k}\right|<r_{0}^{1 / 2},\end{cases}
$$

$q_{0}=-\log r_{0}$.

We shall say that a function in $H^{2}(A)$ is a cyclic vector of $Z_{0}$ if it is contained in no proper doubly invariant subspace of $Z_{0}$. 
Theorem 4. A function $f \not \equiv 0$ in $H^{2}(A)$ is a cyclic vector of $Z_{0}$ if and only if it satisfies the condition

$$
\begin{aligned}
\int_{0}^{2 \pi} \log \left|f\left(r_{0}^{\delta} e^{i t}\right)\right| d t= & \delta \int_{0}^{2 \pi} \log \left|f\left(r_{0} e^{i t}\right)\right| d t \\
& +(1-\delta) \int_{0}^{2 \pi} \log \left|f\left(e^{i t}\right)\right| d t \quad \text { for } 0<\delta<1 .
\end{aligned}
$$

\section{REFERENCES}

1. A. Beurling, On two problems concerning linear transformations in Hilbert space, Acta Math. 81 (1949), 239-255.

2. P. R. Halmos, Shifts on Hilbert spaces, J. Reine Angew. Math. 208 (1961), 102-112.

3. H. Helson and D. Lowdenslager, Invariant subspaces, Proceedings of the International Symposium on Linear Spaces, Jerusalem Academic Press, 1961, pp. 251-265.

UNIVERSITY OF MichigAN

\section{A L C U L US \\ Problems and Solutions}

A. Ginzburg, Technion, Haifa, Israel

Intended (1) to supplement the standard calculus course for students who wish to gain more complete understanding and mastery through analyzing and solving problems of greater variety and difficulty and (2) for those who wish to review their calculus and bring their competence up to a high level. 1200 exercises-with detailed solutions of most of them-are arranged to enable the student to go gradually from the easier to the more difficult. Problems above the level of the ordinary calculus course are included to deepen his insight. Connecting tissue is complete but brief, consisting of short explanations, exact definitions, and basic theorems.

$\$ 7.75$

\section{HOLD EN - D A Y, I N C .} 728 Montgomery St., San Francisco

\section{COLLOQUIUM} PUBLICATIONS

\section{Recent Reprint}

Colloquium 26

GAP AND DENSITY THEOREMS

by Norman Levinson

240 pages $\quad \$ 6.10$ list Price

$25 \%$ discount to members

AMERICAN MATHEMATICAL SOCIETY

190 Hope Street

Providence 6, Rhode Island 